\title{
The utility of ultrasound in the diagnostic evaluation of the posterior ankle joint
}

\section{Wojciech Konarski, Tomasz Poboży}

Department of Orthopedic Surgery, Ciechanów Hospital, Ciechanów, Poland

\begin{abstract}
Sprains are the most common injury of the ankle joint and the most common traumatic injury of the musculoskeletal system. Ultrasound (US) examination of the posterior ankle joint is a challenge for the examiner. This paper focuses on this difficult area and provides guidance on how to effectively perform US examination of the posterior ankle.

Keywords: ultrasound; posterior talofibular ligament; posterior tibiotalar ligament; posterior talocalcaneal ligament; flexor hallucis longus tendon
\end{abstract}

\section{Introduction}

Sprains are the most common ankle injuries and one of the most common traumatic injuries of the human musculoskeletal system, both related and unrelated to sport [1-3]. Various ligaments may be damaged as a result of traumatic injury to the ankle. This paper aims to explore the use of diagnostic ultrasound (US) of the posterior ankle joint, in particular the posterior talofibular ligament and the posterior part of the deltoid ligament (the posterior tibiotalar ligament).

The anterior talofibular ligament is the structure most often damaged in ankle injury [4,5]. Injury of the posterior talofibular ligament is significantly less common and is therefore considered of less clinical importance. However, due to the deep location of this ligament, it is more difficult to assess the condition both via physical examination and via imaging, particularly US examination $[6,7]$. An understanding of the anatomy of this area

Received 09.10.2020 Accepted 30.12.2020

Med Ultrason

2021, Vol. 23, No 2, 226-230

Corresponding author: Wojciech Konarski, MD, PhD

Surgery Clinic, Department of Orthopaedic

Surgery, Ciechanów Hospital,

2 Powstańców Wielkopolskich Street,

06-400 Ciechanów, Poland

E-mail: wkonarski@poczta.onet.pl

Phone: +48 502110863 is key to obtaining good quality images and to facilitate accurate assessment of these structures.

\section{Anatomy of the posterior ankle joint}

When visualizing the posterior ankle point, the posterior part of the tibiotarsal and subtalar joints are visible deep within the joint, particularly the bony contours (fig 1).

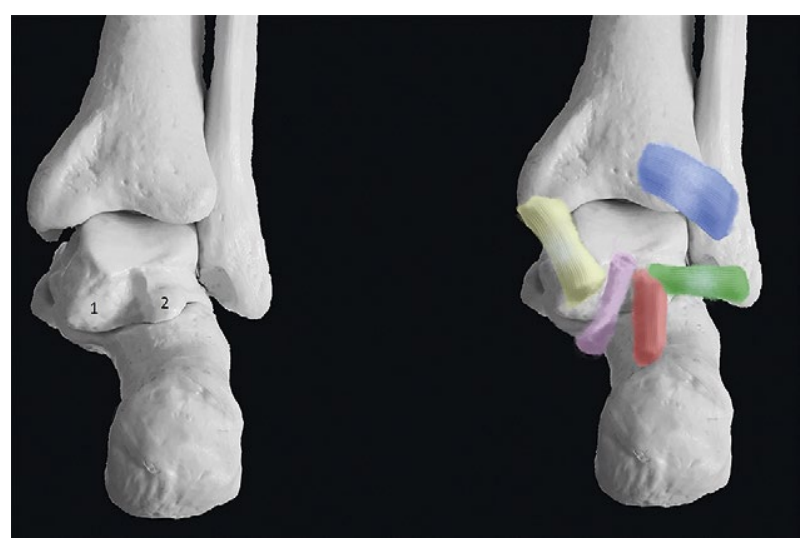

Fig 1. Anatomy of the posterior ankle joint. The posterior process of the talus: the medial (1) and the lateral (2) tubercles. Pink - flexor hallucis longus tendon, green - posterior talofibular ligament), blue - posterior tibiofibular ligament, red - posterior talocalcaneal ligament, yellow - deltoid ligament (posterior tibiotalar part). 



Fig 2. a) US of the distal part of the Achilles tendon, using a longitudinal scan; b) patient and probe positioning. ACH Achilles tendon; K - Kager fad pad; star - retrocalcaneal bursa.

The posterior process of the talus has two tubercles: the medial and the lateral, and accurate imaging of the tubercles is crucial to visualization of the ligaments of the posterior ankle joint. The posterior talofibular ligament attaches to the lateral tubercle, and the posterior part of the deltoid ligament attaches to the medial tubercle. As with most joints, the ligaments provide direct reinforcement of the joint capsule and form the deepest layer of soft tissue, located adjacent to the articular surface of the bones forming the joint. Another important anatomical structure, the flexor hallucis longus tendon, is located in the groove between the two tubercles of the posterior process of the talus, and is important to visualize in the context of diagnostic imaging following ankle injury. Superficial to the joint capsule and ligamentous structures is the connective tissue of the Kager fad pad and more superficial to this is the Achilles tendon. The retrocalcaneal bursa is located between the distal part of the Achilles tendon, the upper surface of the calcaneus and the connective tissue of the Kager fad pad (fig 2).



Fig 4. a) Posterior talofibular ligament (PTFL), longitudinal view and b) probe position. MT - medial tubercle of the posterior process of the talus; LT - lateral tubercle of the posterior process of the talus; star - groove between the two tubercles of the posterior process of the talus (flexor hallucis longus tendon space); arrows - posterior talofibular ligament (PTFL).

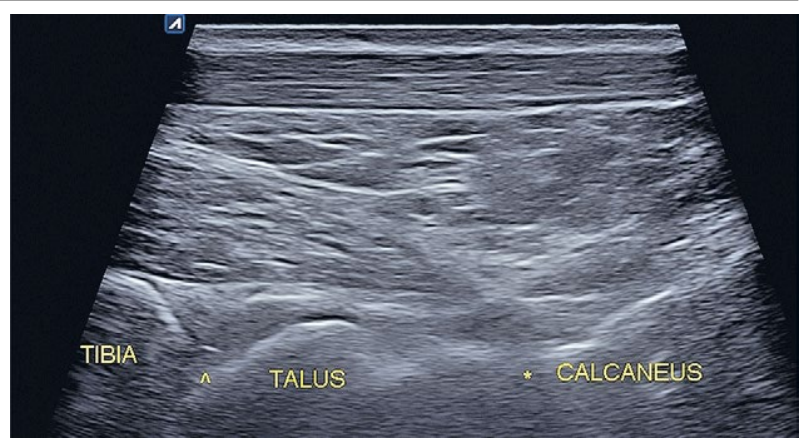

Fig 3. US of the middle part of the Achilles tendon, using a longitudinal probe position. Star - posterior talocalcaneal ligament; arrow - posterior tibiotalar ligament.

\section{US of the posterior ankle joint}

There is uncertainty regarding the most convenient position to perform US of the posterior ankle. The posterior ankle is most accessible when the patient is laying in a prone position with the knee slightly bent and the ankle joint held at an angle of $90^{\circ}$ with the weight of the foot resting on the toes. However, in the case of recent injury, this position may cause considerable pain, so it is often necessary to adapt to the situation and instead examine the patient in a supine position with the knee bent to approximately $90^{\circ}$ and the foot resting on the floor. This alternative position is often sufficient to adequately evaluate the ligaments.

In the first part of the examination, the transducer is placed along the long axis of the limb over the central part of the Achilles tendon. One should remember at the beginning of the examination to set an appropriate depth and focus and to choose an appropriate frequency. A multi-frequency probe with a range of 3-12 MHz, with the frequency set to the upper range is recommended. The goal is to visualize the outline of the bony structures and

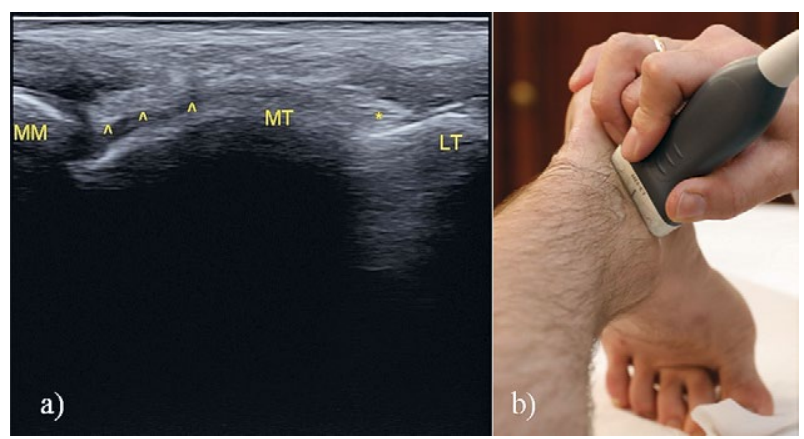

Fig 5. Posterior tibiotalar ligament (part of the deltoid ligament), longitudinal scan; b) patient and probe positioning. MM - malleolus mediualis; MT - medial tubercle of the posterior process of the talus; LT - lateral tubercle of the posterior process of the talus; star - flexor hallucis longus tendon; arrows - posterior tibiotallar ligament. 


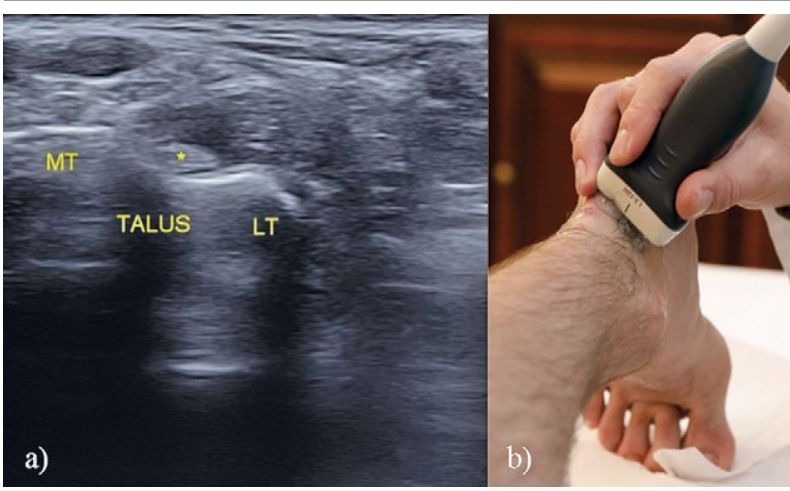

Fig 6. a) Flexor hallucis longus (FHL), transverse scan; b) patient and probe positioning. MT - medial tubercle of the posterior process of the talus; LT - lateral tubercle of the posterior process of the talus; star - flexor hallucis longus tendon.

joint margins, presence or absence of exudate, as well as the presence and size of the os trigonum bone or hypertrophied posterior talus process (fig 3 ).

While the US transducer is positioned along the long axis of the limb, it can be moved medially and laterally whilst remaining in the longitudinal plane, to assess the whole posterior ankle area. This method allows complete visualization of the flexor hallucis longus tendon. This tendon is often easier to initially locate using cross-sectional imaging, where the tendon is visible between the tubercles of the posterior process of the talus.

With the transducer held in the transverse plane, the posterior talofibular ligament and the posterior tibiotalar ligament can be assessed. To fully assess the posterior talofibular ligament, the transducer is placed transversely on the posterolateral aspect of the joint where the posterior surface of the distal end of the fibula and the lateral tubercle of the posterior process of the talus are visible. The ligament appears as the deepest layer of soft tissue, with typical fibrous echostructure adjacent to the bone surface (fig 4).

To assess the posterior tibiotalar ligament (the posterior part of the deltoid ligament) the transducer is placed transversely on the posteromedial aspect of the ankle, where the posterior part of the medial ankle and the medial tubercle of the posterior process of the talus are visible. The posterior tibiotalar ligament appears as the deepest layer of soft tissue located adjacent to the bone surface (fig 5).

With the transducer in the transverse plane the tendon of the flexor hallucis longus is visible in the groove between the tubercles of the posterior process of the talus (fig 6). This can be further assessed in the longitudinal plane (fig 7).

The posterior talocalcaneal ligament is not of significant importance as injury is rare and only tends to occur

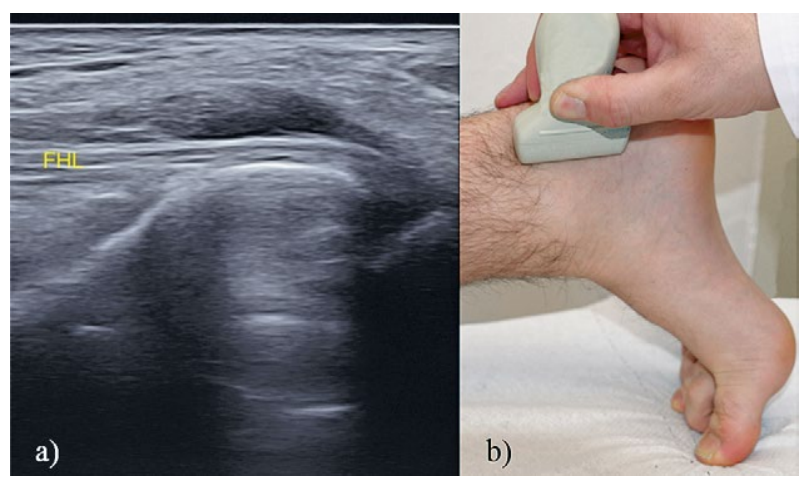

Fig 7. a) Flexor hallucis longus (FHL), longitudinal section; b) patient and probe positioning.



Fig 8. a) Posterior talocalcaneal ligament, longitudinal scan; b) patient and probe positioning (arrows - posterior talocalcaneal ligament).
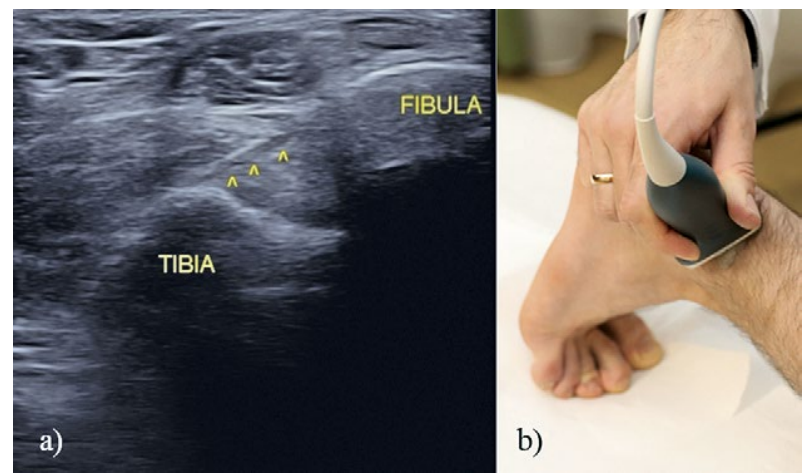

Fig 9. Posterior tibiofibular ligament (posterior part of the tibiofibular syndesmosis); b) patient and probe positioning (arrows - posterior tibiofibular ligament).

in cases of serious injuries. To evaluate this ligament the transducer is held in the longitudinal plane, proximal to the superior margin of the calcaneus. The ligament appears as a fibrous tissue layer connecting the talus and calcaneus (fig 8).

The posterior tibiofibular ligament is located on the posterior surface of the tibiofibular syndesmosis (fig 9). 
To visualize this ligament, the transducer is held in the longitudinal plane slightly proximal to the tibiotalar joint. The ligament is present as a fibrous structure between the posterior tibial process (the most prominent part of the posterior tibia) and the fibula. The tibiofibular syndesmosis should be evaluated from both the posterior and anterior sides, with appropriate comparisons made to the opposite limb.

\section{Pathology of the posterior ankle joint}

US imaging of the ankle joint is often indicated for patients with pain persisting for 4-6 weeks following an injury [8]. However, performing US examination sooner following injury may have a significant impact on the manner and timing of definitive treatment. For some patients, it may reduce the need for unnecessary immobilization and, for others, it may identify damage that requires timely surgical intervention. Typically, ligament damage is assessed in terms of severity using a threepoint grading scale [9].

Grade I injuries occur when the ligament is stretched, but remains intact. Typical symptoms are of local tenderness, with swelling visible on US as a symptom thickening and reduction in echogenicity. In grade II injuries, there is evidence of a partial rupture of the ligament. In recent injuries and up to a few days after the injury, continuity of the ligament may appear preserved, or may display an obvious defect. Grade II injuries are often diagnosed after 2-3 weeks, when scar tissue has started to form. This appears on US imaging as thickening and reduced echogenicity of the ligament, with the thickening being significantly greater than in the case of grade I damage.

In grade III injuries, there is complete rupture of the ligament. Recognition of such damage in the acute setting is straightforward; however, it may be more challenging in the case of chronic injury. Following grade III ligament damage in the ankle, a thin, inefficient scar often forms in the area of the damage, without proper tension of the damaged ligament, either at rest or during functional examination.

Images of ligament damage may vary depending on the location of the damage [8]. Damage along the length of the ligament is easy to evaluate; however, damage to ligament attachment points can present greater diagnostic difficulty. Some ligament attachments may appear to be hypoechogenic, but this does not necessarily indicate damage and rather may suggest anisotropy of the tissue. This often appears at the attachment of the posterior tibial ligament to the fibula. In cases where there is diagnostic doubt, a comparative examination of the other limb, provided this is normal, may be of value. In cases

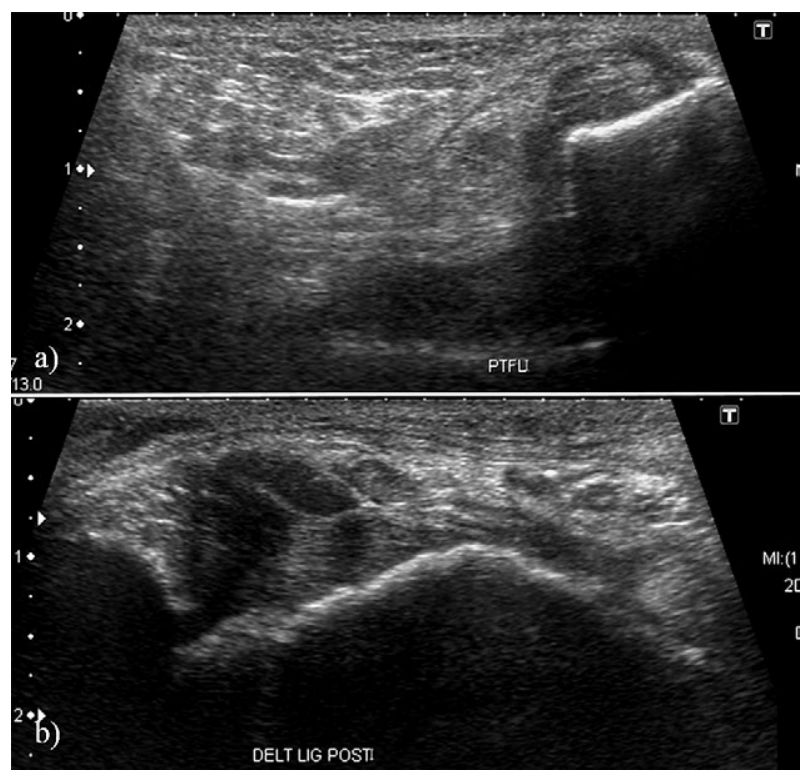

Fig 10. a) Posterior talofibular ligament (PTFL) and b) posterior tibiotalar ligament (part of the deltoid ligament) injuries.

where there is damage to ligament attachments in the ankle joint, it is important to also consider the possibility of avulsion fracture and presence of bone fragments.

Damage to ligaments of the ankle joint, as with other joints, can lead to instability, so functional examination should not be forgotten. The role of functional examination is particularly important in the case of damage to ageing ankle joint ligaments. Joint instability following ligament injury is graded from I to III depending on the degree of joint displacement. Grade I instability is defined as abnormal displacement of bony surfaces that does not exceed 5 millimeters. Grade II instability is defined as displacement between 5 and 10 millimeters. Grade III involves displacement of greater than 10 millimeters, more often seen in cruciate ligament or lateral knee joint injury, but also applicable to some anterior talofibular or calcaneofibular ligament injuries. With regard to posterior ankle joint ligaments, even with complete ligament rupture, it is unlikely that the bones will be displaced more than 5 millimeters apart.

The degree of joint instability does not necessarily coincide with the degree of damage to the ligament. Grade I and most grade II ligament injuries do not result in measurable instability. Ankle instability is usually the result of grade III damage. The extent of instability is also related to the manner in which it is healed, which in turn is a result of the treatment applied. Clinical practice has shown that US examination is reliable for assessing grade I and grade II damage (fig 10); however, MRI appears to be more effective in the assessment of grade III damage [10-13]. 


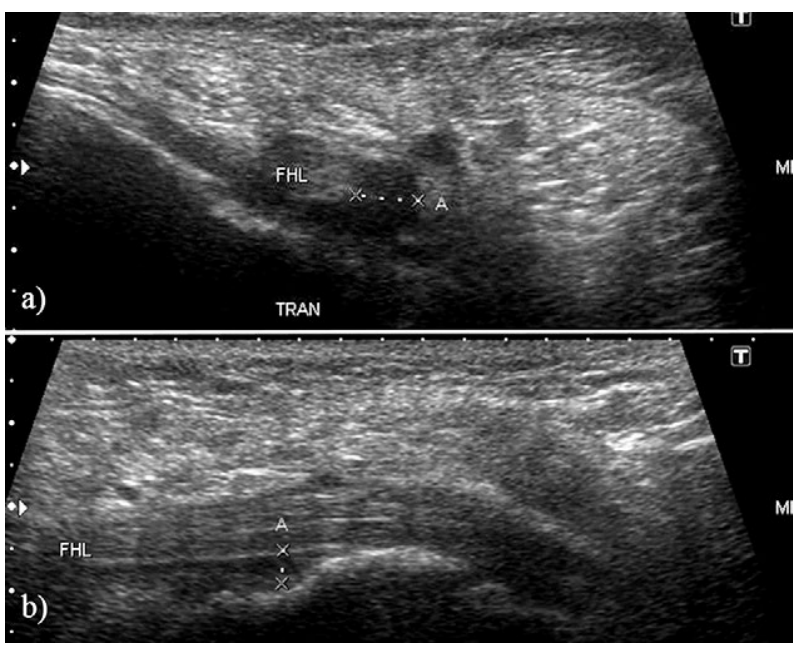

Fig 11. Flexor hallucis longus (FHL) inflammation, transverse (a) and longitudinal (b) scan $\left(\mathrm{X}^{*} \mathrm{X}\right.$ and $\mathrm{A}$ - edema around the tendon and its dimension)

Post-traumatic lesions of the flexor hallucis longus tendon are rare (fig 11). The most frequently observed pathology is tenosynovitis (fig 12), often associated with posterior ankle impingement syndrome, which is caused by the presence of a large os trigonum bone. The medial surface of the bone may adhere to the tendon sheath and as a result of mechanical irritation, cause exudative tenosynovitis This condition most often occurs in athletes such as footballers or ballet dancers and is associated with frequent flexion of the ankle [11].

\section{Conclusion}

US is a valuable modality to assess the structures within the posterior ankle joint. The main utility is in the diagnostic imaging of the posterior talofibular ligament and the posterior portion of the deltoid ligament following ankle injury. US is also useful in the evaluation of posterior ankle impingement syndrome caused by the presence of a hypertrophied os trigonum bone, which can cause an exudative flexor hallucis longus tenosynovitis. US also provides relatively low cost diagnostic imaging following ankle trauma in real time.

\section{References}

1. Polzer H, Kanz KG, Prall WC, et al. Diagnosis and treatment of acute ankle injuries: development of an evidencebased algorithm. Orthop Rev (Pavia) 2012;4:e5.

2. Boruta PM, Bishop JO, Braly WG, Tullos HS. Acute Lateral Ankle Ligament Injuries: A Literature Review. Foot Ankle 1990;11:107-113.

3. Fong DT, Man CY, Yung PS, Cheung SY, Chan KM. Sportrelated ankle injuries attending an accident and emergency department. Injury 2008;39:1222-1227.
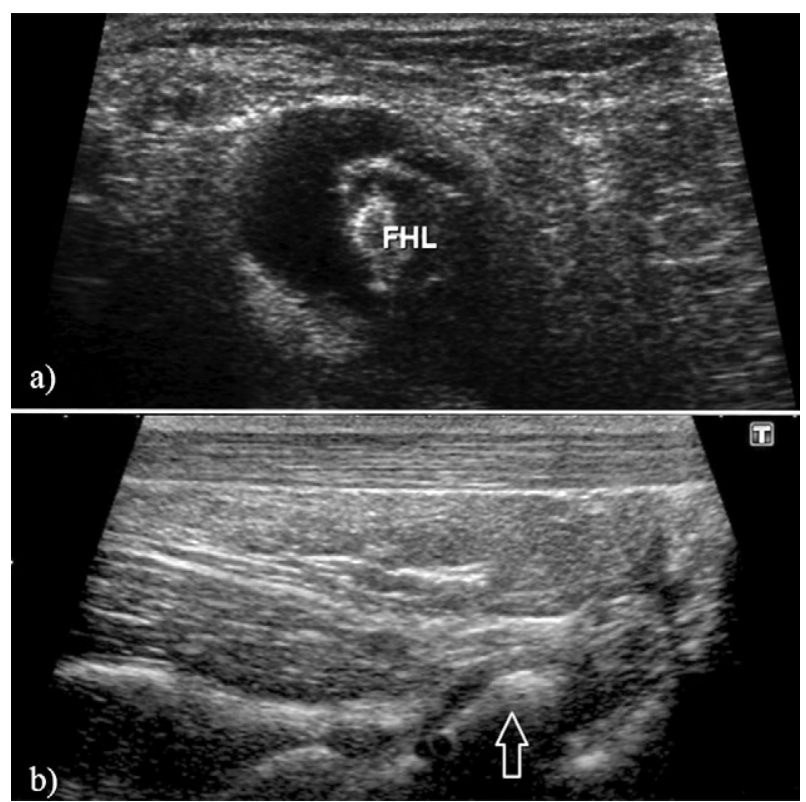

Fig 12. Flexor hallucis longus (FHL) tenosynovitis, transverse scan (a) due to the presence of os trigonum (arrow) (b).

4. Fursdon T, Platt S. The incidence and significance of posterior talofibular ligament injury on magnetic resonance imaging. Orthop Proc 2018;94B SUPP_XXII:56.

5. Kumai T, Takakura Y, Rufai A, Milz S, Benjamin M. The functional anatomy of the human anterior talofibular ligament in relation to ankle sprains. J Anat 2002;200:457465 .

6. Arthurs G, Nicholls B. Ultrasound in anesthesia, critical care and pain management. Cambridge University Press 2016.

7. Park JW, Lee SJ, Choo HJ, Kim SK, Gwak HC, Lee SM. Ultrasonography of the ankle joint. Ultrasonography 2017;36:321-335.

8. Fessell DP, Vanderschueren GM, Jacobson JA, et al. US of the ankle: technique, anatomy, and diagnosis of pathologic conditions. Radiographics 1998;18:325-340.

9. George J, Jaafar Z, Hairi IR, Hussein KH. The correlation between dynamic ultrasound evaluation and clinical laxity grading of ATFL and CFL tears among athletes. J Sports Med Phys Fitness 2020;60:749-757.

10. Margetic P, Salaj M, Lubina IZ. The Value of Ultrasound in Acute Ankle Injury: Comparison With MR. Eur J Trauma Emerg Surg 2009;35:141-146.

11. Yasui Y, Hannon CP, Hurley E, Kennedy JG. Posterior ankle impingement syndrome: A systematic four-stage approach. World J Orthop 2016;7:657-663.

12. Bianchi S, Martinoli C, Gaignot C, De Gautard R, Meyer JM. Ultrasound of the Ankle: Anatomy of the Tendons, Bursae, and Ligaments. Semin Musculoskelet Radiol 2005;9:243-259.

13. Khoury V, Guillin R, Dhanju J, Cardinal É. Ultrasound of Ankle and Foot: Overuse and Sports Injuries. Semin Musculoskelet Radiol 2007;11:149-161. 